\title{
> RESEARCH IN BRIEF
}

\section{The genomic landscape of malaria mosquitos in Africa}

Malaria continues to be one of the top killers in Africa, but scientists are hopeful that new techniques, such as gene-drive systems based on CRISPR/Cas9, may help lead to the eradication of malaria-carrying mosquitos. But to make best use of new genetic tools, researchers will have to know more about the variability in mosquito populations at the genomic level.

To this end, researchers in the Anopheles gambiae 1000 genomes Consortium report the sequencing of 765 different specimens of Anopheles gambiae across 15 locations in Africa (Nature 552, 96-100; 2017). Their data reveal a complex genomic landscape with significant amounts of diversity that may need to be taken into account when developing future efforts to control Anopheles gambiae populations and control the spread of malaria.

\section{A CRISPR/Cas9 system to turn genes on in vivo}

Novel methods for genome editing, particularly CRISPR/Cas9, have opened up new avenues in research and therapy development, owing to the ease, accuracy, and efficiency of their use to manipulate genes. Although originally discovered and developed for efficient and accurate cutting of genes (via double-strand breaks), the CRISPR/Cas9 system has also recently been adapted to help turn genes on. Just like cutting out or modifying disease-causing alleles can help treat certain genetic disorders, upregulating specific genes could have significant therapeutic benefits. However, the modifications to Cas9 required for efficiently turning genes on makes it too large to be encoded within viral vectors typically used to deliver payloads to animals in vivo. In a new paper published in Cell, senior author Juan Carlos Izpisua Belmonte and colleagues have developed a CRISPR/Cas9 system small enough to fit into a viral package (adeno-associate virus) for delivery in vivo, but with all the necessary elements needed for efficient and targeted gene manipulation (Cell 171, 1495-1507; 2017).

In their report, the team tested their new system on several mouse models of human disease, to assess the method's potential to alter phenotypes, and found phenotyping improvements for mouse models of diabetes, muscular dystrophy, and acute kidney disease. Overall, the results represent an important proof of concept for further developing CRISPR/ Cas9 into a epigenome regulating system that could have significant impact on future therapeutic development.

\section{Maternal age linked to variation in worm phenotypes}

It is a well-established fact that individuals who are genetically identical and grow in the same environment can express substantially different phenotypic traits. What is less well-known is why. In a new report by Ben Lehner and colleagues from the Barcelona Institute of Science and Technology, the Universitat Pompeu Fabra, and the Institució Catalana de Recerca (Barcelona, Spain), they describe the role that maternal age and a specific group of genes controlling yolk provisioning can play on the outcomes of progeny (Nature 552, 106-109; 2017).

After establishing that a number of progeny phenotypes (slow development, germline acceleration, brood size etc.) were significantly associated with the age of the offsprings' mothers, the team sought to identify a potential molecular mechanism. Using a number of genetic and imaging methods, the team identified a group of genes encoding yolk proteins that could explain the phenotypic variation with maternal age. They found that as a mother worm ages, the provisioning of yolk to embryos increasing, giving the offspring a jumpstart on several phenotypes that the children of younger mothers missed out on. But, the researchers note, there is an important evolutionary fitness balance that the worms have to contend with: younger mothers, although providing less yolk for their offspring, have the benefit of having children sooner and avoiding other problems that can occur as aging increases.

Overall, the researchers conclude that maternal age (and expression of vitellogenin genes) is a significant variable impacting the phenotypic variation in future generation of worms, adding important mechanistic knowledge explaining potential strategies for animal fitness, but also for reproducible outcomes in the lab. 


\section{Cracking the bad egg}

When zebrafish spawn, some eggs will develop into healthy larval fish while others won't. A new paper takes a closer look at what separates good eggs from bad (PLoS One 12, e0188084; 2017). To define those categories, the researchers froze eggs sampled from batches produced by $A B$ strain zebrafish and then watched as the remainder continued to grow. Batches with $>90 \%$ survival 24 hours post-hatching were considered "good quality", while those with $<30 \%$ survival during the same period were considered "poor." They then ran proteomic analyses on the frozen eggs. Poor quality eggs lacked proteins necessary for proper metabolism and protein synthesis, while having too many proteins typically involved in cell degradation. Overall, the researchers identified 17 protein markers that they think may be indicative of egg quality.

\section{Dead-reckoning bats}

At dusk, a bat leaves its roost and heads off towards its foraging site. It takes advantage of a flyway - a long, linear stretch, like a river or forest edge-but how does it know when to turn? Perhaps with the help of landmarks, or by using acoustic flow to gauge distance traveled? According to Yossi Yovel's bat lab, those ideas are dead wrong: they suggest bats use internal path recognition, or dead reckoning, to navigate flyways (Curr. Biol. 27, 3650-3657; 2017).

The lab trained Kuhl's pipistrelles to fly down a corridor to a platform. They then removed the platform and modified the corridor to test the different navigational possibilities. Changing landmarks didn't confuse the bats, nor did disrupting the acoustics in the corridorthey still hovered were they expected the platform to be. But messing with the bats' internal senses, like adding a small weight to their backs, was enough to throw them off.

\section{Doxycycline \& dysbiosis}

Doxycycline is a common effector used to control gene expression in transgenic mice. It's also an antibiotic, with the potential to at least temporarily alter an organism's microbiome. Researchers at the University of Michigan Medical School's Unit for Laboratory Animal Medicine recently took what they believe to be the first look at doxycycline's effect on mice (BMC Res Notes 10, 644; 2017). Though it was a small study comparing the fecal microbiota of female $\mathrm{C} 57 \mathrm{BL} / 6 \mathrm{NCrl}$ mice that received drinking water doses of the antibiotic over 4 weeks to those that didn't, the results suggest that doxycycline does make a difference: treated mice had decreased microbial richness and diversity and changes in relative abundance of different bacterial species compared to controls. Just one more variable to be aware of when using doxycycline-induced models, the authors suggest.

\section{Wnt-less complexity}

For multicellular organisms to develop, their cells need ways to communicate. In animals, the Wnt signaling pathway is one of three (along with Notch and TGF- $\beta$ ) thought to be necessary for proper development. But a new study in a very basal animal suggests otherwise (Sci. Rep. 7, 15383; 2017).

Poriferans may not exactly look alive, but they are considered part of the animal kingdom. But one particular class, the glass sponges, are a bit different. Rather than forming distinct cells, glass sponges are primarily made up of syncytial tissues, multinucleated masses of cytoplasm, linked to other cell types by plugged junctions. Genetic comparison of two different glass sponges to other metazoan animals revealed an absence of genes in the Wnt pathway, although genes from the other two pathways were conserved. Glass sponges' syncytial organization may have made Wnt irrelevant. 